\title{
DER ZWEITE THORNER FRIEDEN (1466) IN DER POLNISCHEN UND PREUSSISCHEN POLITISCHEN ARGUMENTATION DES r6. JAHRHUNDERTS
}

Die bekannte rechtshistorische Diskussion um die Lehnsproblematik des am 19. Oktober 1466 zwischen König Kasimir IV. von Polen und Deutschordens-Hochmeister Ludwig von Erlichshausen geschlossenen Zweiten Thorner Friedens ${ }^{1}$, an welcher der gefeierte Jubilar, Professor Adam Vetulani, entscheidenden Anteil hat ${ }^{2}$, könnte eine neue Perspektive gewinnen, wenn es gelänge, sie an ihren Ursprungsort zurückzuverfolgen; eine präzise wissenschaftliche Analyse des historischen Selbstverständnisses des Zeit nach 1466 bleibt jedoch, trotz vorhandener Ansätze ${ }^{3}$, immer noch ein Postulat.

Untersuchungen des Verfassers zum preußisch-polnischen Lehnsvernältnis ${ }^{4}$ nach dem für die deutsch-polnische Symbiose im Nordosten Europas entscheidenden nächsten politischen Ereignis, dem zwischen

1 Hier sei für die bis zum 500. Jahrestag des Thorner Friedens erschienenen Arbeiten verwiesen auf die Bibliographie von Janina Huppenthal: II pokó $j$ toruński. Poradnik bibliograficzny, Torun 1966. Danach vor allem Marian Biskup: Traktat toruński 1466, Bydgoszcz 1966; Karol Górski: Pokój toruński 1466 roku i jego znaczenie dla Polski, Warszawa 1966; sowie Wojciech Hejnosz: Traktat toruński $z 1466$ r. $i$ jego prawnopolityczne znaczenie, in: Zapiski Historyczne 31 (1966) 91-108, sowie derselbe: Der Friedensvertrag von Thorn (Toruń) 1466 und seine staatsrechtliche Bedeutung, in: Acta Poloniae historica 17, 1966, 105-122.

2 Das Thorn-Problem erscheint im Gesamtwerk von Adam Vetulani immer wieder. Hier sei vor allem die minutiöse Analyse des Thorner Vertrags und dessen rechtshistorischer Vergleich mit dem Krakauer Frieden hervorgehoben, in: Adam Vetulani, Lenno Pruskie (Od traktatu krakowskiego do śmierci księcia Albrechta 1525-1568). Studjum historyczno-prawne, Kraków 1930, 1-123 passim.

3 Siehe die im Zusammenhang mit der vorliegenden Fragestellung nicht immer befriedigende Studie von Erich Weise: Die Beurteilung des Zweiten Thorner Vertrags von 1466 durch die Zeitgenossen bis zum Ende seiner Rechtswirksamkeit im Jahre 1497, in: Zeitschrift für Ostforschung 15 (1966) 601-621.

${ }_{4}$ Stephan Dolezel: Das preußisch-polnische Lehnsverhältnis unter Herzog Albrecht von Preußen, Köln-Berlin 1967. 
König Sigismund I. und seinem Neffen, dem aus dem Hochmeisteramt scheidenden Markgrafen Albrecht von Brandenburg-Ansbach geschlossenen Krakauer Frieden vom 8. April $1525^{5}$, ergaben am Rande, daß der Rechtscharakter des Zweiten Thorner Friedens von den polnischen und preußischen Akteuren des 16. Jahrhunderts je nach der aktuellen politischen Intention manipulierbar erschien. Ihre Argumentation, in diesem Zusammenhang bislang nicht publiziert ${ }^{6}$, dürfte für die oben erwähnte Diskussion nicht ohne Interesse sein.

Die polnische Diplomatie hatte nach der im Krakauer Frieden fixierten Umwandlung des Ordensterritoriums in ein weltliches, von der Krone Polen lehnsabhängiges erbliches Herzogtum naturgemäß zumindest mit päpstlichen und kaiserlichen Protesten, wenn nicht gar mit konkreten militärischen Konsequenzen zu rechnen. Es ist daher nicht verwunderlich, daß sie bestrebt war, die revolutionäre Bedeutung des Krakauer Ereignisses zu verschleiern ${ }^{7}$. So erhielt der königliche „orator perpetuus” am Kaiserhof, Johannes Dantiscus, nachdem bezeichnenderweise vorerst ein Vierteljahr ohne jede klärende Instruktion verstrichen war, in einem am 11. Juni 1525 abgefertigten königlichen Memorial ${ }^{8}$ lediglich die Anweisung, Karl V. davon zu überzeugen, daß Sigismund an der konfessionellen Änderung im neuen Herzogtum keine Schuld trage: In Krakau sei ,,über die Religion [...] nichts verhandelt worden”, da dies „,nicht unsere Aufgabe, noch wir die Institutoren des Ordens; schließlich da es im ganzen Ordensland um den Katholizismus bereits geschehen war". Sigismund habe lediglich ,die Gelegenheit, welche die verderbte Zeit uns anbot, für die Sache des Friedens genutzt, welchen man auf andere Weise nicht erlangen konnte". In welcher Weise notfalls die politische Seite des Krakauer Akts zu interpretieren war, geht aus einem Handschreiben Sigismunds an Papst Klemens VII. vom 20. Mai 1525 hervor 9. Der König stellte die Vorgänge so dar, als habe er mit Albrecht nicht etwa einen neuen Vertrag geschlossen, sondern nur einen alten bekräftigt. Der einstige Hochmeister habe der Krone gegenüber nur ,,seine Lehenspflicht" geleistet, welche ihm abzufordern der König (unausgesprochen: gemäß dem Zweiten Thorner Frieden) ,,berechtigt” gewesen sei.

5 Stephan und Heidrun Dolezel: Die Staatsverträge des Herzogtums Preußen, Teil 1: Polen und Litauen, Köln und Berlin 1971, $12 \mathrm{ff.}$

${ }_{6}$ Kurze thesenartige Zusammenfassung der hier vorgelegten Argumente des Verfassers in: Preußenland, Marburg 1966.

7 Zur Entwicklung der polnischen Verteidigungskonzeption gegenüber Kaiser und Papst siehe Dolezel, Lehnsverhältnis, 32ff.

8 Acta Tomiciana VII, Nr 49.

91525 Mai 20, [Krakau], Sigismund an Klemens VII., Matthias Dogiel: Coäex diplomaticus regni Poloniae et Magni Ducatus Lituaniae, IV, Wilno 1764, 236, dt. bei J. Vota [d. i. Onno Klopp]: Der Untergang des Ordensstaates Preußen und die Entstehung der preußischen Königswürde, Mainz 1911, 333. Vgl. a. Acta Tomiciana VII, Nr 35. 
- Es war kein geringerer als Johannes Dantiscus, der die hier erstmalig festzustellende Taktik in den nächsten Jahren zum Prinzip der diplomatischen Verteidigung des Krakauer Friedens erhob: In der Gleichsetzung des Krakauer Traktats mit dem Zweiten Thorner Frieden wurde das Moment der politischen Neuerung verschwiegen und der polnische König dadurch, daß man nur die religiös e Neuerung als das einzig echte Novum des Friedens von 1525 herausstellte, in seiner Verantwortung entlastet. Dantiscus, der sich in den Jahren 1524-1532 während seiner dritten Gesandtschaftsreise oft im Kreise der engsten Begleiter Karls V. bewegte und zeitweilig als kaiserlicher Vertrauter galt, wußte nicht nur bald nach Polen zu berichten, daß sich der Kaiser mit dem preußischen Problem durchaus ,widerwillig" befaßte ${ }^{10}$. Der polnische Diplomat mutete dem im Ausland erzogenen Karl offenbar auch keine allzu genaue Sachkenntnis in Polonicis bzw. Prussicis zu, wie man seinen an den Kaiser adressierten historischen Exkursen entnehmen darf ${ }^{11}$ : Preußen, das schon immer zu Polen gehört habe, sei einst während eines jahrelangen polnischen Interregnums von ,einem Herzog von $\mathrm{Ma}-$ sowien [...] usurpiert" worden. Da der Masowier das Land aber nicht aus eigener Kraft habe völlig unterwerfen können, sei er gezwungen gewesen, den Deutschen Orden nach Preußen zur Hilfe zu rufen; diesem habe er Land geschenkt, das ihm selbst ,nicht gehörte”. Fortan habe sich der Orden geweigert, das Land an Polen als den einzig rechtmäßigen Besitzer zurückzugeben. Nachdem der Orden seine Missionsaufgabe verloren hatte, weil jedermann getauft worden sei, habe er sich zum dauernden Störenfried gegenüber Polen entwickelt. König Sigismund, angesichts der Türkengefahr um die Friedenssicherung im Norden bestrebt, habe schließlich dem letzten Hochmeister Preußen zum Lehen gegeben ,auf Grund der Macht und des oberhoheitlichen Rechts”, wobei er von ihm nichts anderes gefordert habe, als die ,geschuldete Huldigung”, die schon vor ihm ,einige Hochmeister" geleistet hätten.

Es war die große Supplik des Deutschen Ordens vom 25. Juni $1530{ }^{12}$, welche beim Augsburger Reichstag desselben Jahres Dantiscus' Hoffnungen auf die Überzeugungskraft seiner Darstellungsweise zerstören sollte. Die - freilich sehr theoretische - kaiserliche Belehnung des Deutschmeisters Walter von Kronberg mit Preußen am 26. Juli 1530, die Annullierung des Krakauer Friedens durch Karls V. Mandat vom 14. November 1530 und die Ächtung des preußischen Herzogs vom 19. Januar 1532

10 Urteil über Karls Haltung: 1526 Dezember 6, Granada, Dantiscus an Sigismund, Acta Tomiciana VIII, Nr 258.

11 Gesandtschaftsbericht Dantiscus' an Sigismund, 1530 Juli 29, Augsburg, Acta Tomiciana XII, Nr 213 (Hiervon erhielt der Herzog eine Abschrift durch Szydłowiecki, vgl. Acta Tomiciana XII, Nr 218).

12 1530 Juni 25, Supplik Kronbergs: Text bei Vota, 358ff. 
waren die Quittung für die in diesem Punkt verfehlte Politik des berühmten ermländischen Bischofs.

Dennoch schritt die polnische Kanzlei, auf deutschen Reichstagen mit ihren Argumenten immer wieder scheiternd, auf dem einmal eingeschlagenen Weg fort. Als sich Herzog Albrecht beim polnischen Winterreichstag 1530 gegen die polnische Art der Verteidigung des Krakauer Friedens wandte, mußte er erfahren, daß sein Lehnsherr nicht bereit war, eine andere Position zu beziehen. Der Herzog werde, so hieß es in der Anwort Sigismunds ${ }^{13}$, von seinen Feinden aus zweierlei Gründen angegriffen: einerseits wegen des Lehnseides, andererseits wegen des Abfalls von Papst und Orden. Nur der Vorwurf der „religiösen Änderung” sei ernst zu nehmen, weswegen man dem Herzog (ungeachtet dessen, daß er die Ordensbesitzungen längst säkularisiert und selbst durch seine im Juli 1526 geschlossene Ehe mit der dänischen Prinzessin Dorothea den Zölibat gebrochen hatte) raten müsse, in den Schoß der römischen Kirche zurückzukehren. Der Vorwurf aber, eine neue Lehnsbindung eingegangen zu sein, treffe den Herzog zu Unrecht. Es sei „dem ganzen Erdkreis bekannt”, daß Polen 1466 ,die preußischen Lande als seine Erblande rechtmäßig und mit kriegerischer Gewalt erobert" und den Hochmeister der „Herrschaft und dem Lehnsdienst [sic!] unserer Vorfahren unterworfen” habe. Albrecht habe folglich um „des Friedens und des Staatswohles willen das getan [...], wozu Euer Durchlaucht überhaupt und durch den Thorner Frieden verpflichtet war und was die meisten Vorgänger Eurer Durchlaucht getan haben"; eine solche Tat könne somit auch ,niemandem als unwürdig oder neuartig erscheinen”.

Allen preußischen Änderungswünschen zum Trotz gehörte auch in Zukunft die These, der Krakauer Frieden unterscheide sich vom Thorner Frieden lediglich dadurch, daß sich Albrecht zur Annahme der lutherischen Konfession entschloß, zur Grundlinie der defensiven polnischen Diplomatie und Publizistik. So verteidigte der polnische Gesandte Stanisław Maciejowski, auf Dantiscus' Formulierungen zurückgreifend, die Ereignisse des Jahres 1525 vor dem Regensburger Reichstag $1541^{14}$ : Albrecht habe sich unter polnische Lehnshoheit begeben, ,zu welcher [...] auch die alten dieses Ordens Hochmeister, ehe und zuvor sich etliche unter ihnen widerwärtig zu sein unterstanden, [verpflichtet] gewesen sein". Den Herzog treffe die seit 1532 vom Kaiser immer wieder

131531 Januar 8, Petrikau, Antwort Sigismunds an Albrecht, Staatsarchiv Königsberg (= Staatliches Archivlager Göttingen). Herzogliches Briefarchiv (fortan HBA), B 1, 295; Acta Tomiciana XII, Nr 10 und XIII, Nr 17; W. Hubatsch, Europäische Briefe im Reformationszeitalter, Kitzingen 1949, $23 \mathrm{f}$.

141541 o T. [April?], „Instruktion K. Mt. zu Poln” an St. Maciejowski, mehrere Konzepte und Reinschrift: HBA H 774. 
verhängte Reichsacht nicht rechtens, habe doch der einstige Hochmeister nur ,getan, was ihm zuständig gewest”.

Der junge Stanisław Łaski, als königlicher Orator 1548 nach Augsburg gesandt, erklärte den deutschen Ständevertretern und dem Reichsregiment ${ }^{15}$, der Deutsche Orden sei von allem Anfang an Polen untertan gewesen, habe später widerrechtlich seine Waffen gegen den polnischen Oberherrn gekehrt, bis er im Zweiten Thorner Frieden gezwungen worden sei, „dem König von Polen undertänig und getreu zu sein, wie von alters her". Obwohl Ludwig von Erlichshausen eben dies 1466 beeidigt habe, seien doch seine Nachfolger, schließlich auch Albrecht, von Polen wieder ,abgefallen” und hätten gegen ihre „Lehnsherren” wieder Krieg geführt. „Aus dem Willen und Straf Gottes” sei Albrecht endlich so geschwächt worden, daß er sich 1525 in Krakau zum Frieden gefügt habe, wie es einem "frommen getreuen Lehnsmann” zustehe. Preußen gehöre „über Menschengedächtnus” zu Polen; es ,,anderer Gerechtigkeit" unterstellen zu wollen, wie es der Deutschmeister jetzt und hier fordere, sei ,unredlich".

Dem Wort des polnischen Vertreters war auch diesmal, ebenso wie in allen späteren Fällen (polnische Gesandte bemühten sich in den nächsten zwei Jahrzehnten mit ähnlichen Argumenten wiederholt um die Aufhebung der über Albrecht ausgesprochenen Reichsacht) wenig Überzeugungskraft vergönnt. Immer wieder brachte der Orden die deutschen Reichstage zu der Feststellung, daß die Darlegungen der Gegenseite „,nicht von dem rechten und wahren ursprünglichen Anfang der Geschichten" seien ${ }^{16}$. Nicht den historischen Traktaten eines Johannes Dantiscus und seiner Nachfolger, sondern günstigen politischen Umständen die hier nicht dargelegt werden sollen ${ }^{17}$ - hatte es der Herzog zu verdanken, daß die Reichsacht mehrfach suspendiert wurde; eine völlige Aufhebung des kaiserlichen Bannspruchs vermochte Polen bekanntlich bis zum Tode Albrechts (1568) nicht zu erreichen.

Die Furcht vor einer drohenden Reichsexekution, die sich des Herzogtums Preußen nach der 1527 erfolgten Erhebung des Deutschmeisters Walter von Kronberg zum Administrator des Hochmeisteramts in zunehmendem Maße zu bemächtigen begann, drängte auch die preußische politische Publizistik in die Nähe polnischer Argumente, ja es gibt sogar Anzeichen dafür, daß der herzogliche Gesandte Georg Klingenbeck

151548 o. T. [vor Januar 23], Rede Easkis auf dem Reichstag zu Augsburg: HBA H 792 und 794; Dogiel IV, 314-318; Easki änderte hierbei leicht die Instruktion des Königs; letztere in: HBA Konzepte H 1270 und Staatsarchiv Königsberg, Ostpreußischer Foliant 100, fol. 141ff.

${ }_{16} 1548$ o. T., Reichstag Augsburg, Entgegnung des Deutschmeisters, HBA H 792; Dogiel IV, 318-323.

${ }_{17}$ Zur Suspension der Reichsacht siehe Dolezel, Lehnsverhältnis, 54ff. 
zu ähnlichen Sprachregelungen riet, wie sie Dantiscus 1530 in Augsburg vertreten hatte ${ }^{18}$. Albrecht selbst sprach in seiner großen Apologie des Jahres $1531{ }^{19}$, durchaus im Sinne seines Lehnsherrn, von berechtigen polnischen Forderungen, die an ihn während seiner Hochmeisterzeit gestellt worden seien. Doch ging der Herzog nicht so weit, den Krakauer Vertrag mit demjenigen von Thorn gleichzusetzen, sondern legte vielmehr dar, daß der. König ihn 1525 vor die Alternative gestellt habe, die Friedensbedingungen von 1466 anzunehmen oder sich mit dem Herzogtum belehnen zu lassen.

$\mathrm{Zu}$ einer entschiedenen Zurückweisung der polnischen These, die preußische Bindung an Polen sei nach 1525 essentiell die gleiche geblieben, die sie vorher war, entschloß sich der Herzog aus einem anderen Anlaß. Bei der Krönung Sigismunds II. August zum König von Polen, die 1530, zu Lebzeiten seines Vaters, nach einer von der Königinmutter Bona manipulierten überstürzten Königswahl stattfand ${ }^{20}$, protestierte Albrecht dagegen, daß er, obwohl ihm im Artikel 14 des Krakauer Vertrags die höchste Senatorwürde in Polen (der ,erste Platz nächst dem König") eingeräumt wurde, nicht zum Wahlkonvent geladen worden war, und verlangte einen entsprechenden Rechtsvorbehalt, der sein Wahlrecht für alle künftigen Fälle absichern sollte. Die königliche Kanzlei jedoch weigerte sich, ein Wahlrecht des Herzogs anzuerkennen, mit dem Hinweis, es sei im Vertrag nicht wörtlich (nominatim) verankert. Die Krone behauptete stattdessen, man könne aus der bloßen Senatorwürde in Polen kein Wahlrecht ableiten, weil angeblich auch die Hochmeister, seit 1466 Senatoren der Krone, das Recht zur Teilnahme an polnischen Königswahlen nicht besessen hätten. Der Herzog, ihr unmittelbarer Rechtsnachfolger, müsse sich daher mit derselben ,senatoria potestas" des Jahres 1466 begnügen, die das Wahlrecht nun eben nicht einschließe. Albrecht war es offenbar nicht bekannt, daß die Krone, wie Adam Vetulani 1930 nachwies ${ }^{21}$, nach dem Tode König Kasimirs den Hochmeister Hans von Tiefen zur Königswahl geladen hatte, das polnische Argument also schon aus diesem Grunde nicht stichhaltig war. Der Herzog verwahrte sich vielmehr mit aller Entschiedenheit ${ }^{22}$ gegen den Versuch, seinen Status mit demjenigen der Hochmeister nach 1466 zu vergleichen. Das Ordensoberhaupt habe nach dem Zweiten Thorner Frieden, so ließ Albrecht durch

18 Dolezel, Lehnsverhältnis, 50f.

19 Apologie, 1531 Mai 31, HBA H 758, 77 Folio; siehe auch Kurt Forstreuter: Vom Ordensstaat zum Fürstentum, Kitzingen 1951, $147 \mathrm{ff}$.

20 Hierzu ausführlicher: Stephan Dolezel: Herzog Albrecht und die polnische Königswahl, in: Zeitschrift für Ostforschung 13, (1964) 478-489. Siehe auch Dolezel, Lehnsverhältnis, $117 \mathrm{ff}$.

21 Vetulani, Lenno, 151. Auch im letzten gültigen Wahldekret (1506) waren die Hochmeister nicht vom Wahlrecht ausgenommen.

${ }_{22} 1530$ [März], Reichstag Krakau, Antwort Albrechts, Ostpreußischer Foliant 92, fol. $227 \mathrm{~h}$ ff. 
seinen Vertreter antworten, ,als ein Bedrungener nicht mehr denn [seine] Ratspflicht getan, auch der Kron Poln nie undertänig sein wollen. Mein gnädigster Herr, der Herzog aber sei ein Lehns- und undertäniger Fürst, der sich ganz und gar [zu] einem Poln gemacht, die Lehen offentlich entpfangen, auch mit allen Ständ der Kron zu Poln Guts und Bös leiden muß, und will Leib, Gut, Plut und alles Seiner. Fürstlich Gnaden Vermögen, aus schuldiger Pflicht bei der löblichen Kron Poln lassen". Damit war die herzogliche Auffassung vom Unterschied zwischen der Thorner und der Krakauer Bindung an Polen in prägnanter Weise hervorgehoben: Während der Deutschordens-Hochmeister Ludwig von Erlichshausen als Besiegter in die Fesseln des Thorner Friedens gezwungen wurde, war der Brandenburger - so mochte er es zumindest verstanden wissen - aus freien Stücken den Krakauer Vertrag eingegangen. Die Hochmeister taten nur ihre Ratspflicht, der Herzog aber fühlte sich als Lehnsmann an das Geschick der Krone gebunden und war, wie er es selbst nicht ohne Pathos darzustellen verstand, bereit, für die gemeinsame Sache einzustehen. Die Kronkanzlei setzte sich freilich über die herzogliche Interpretation der Rechtslage hinweg, das Wahlrecht blieb Albrecht zeitlebens versagt.

$E_{S}$ kennzeichnet allerdings die Wendigkeit der polnischen Kronjuristen, wenn der Nachfolger Sigismunds, Sigismund August, wenige Jahre später ein anderes Rechtsbegehren ${ }^{23}$ des Herzogs mit einem aus seinem Mund wahrhaft erstaunlich klingenden Argument zurückwies: Der Artikel 20 des Krakauer Friedens bestimmte, daß Einwohner des Herzogtums vor kein fremdes Gericht gezogen werden dürften. Aus diesem Vertragspassus leitete der Herzog mit vollem Recht sein generelles ,privilegium de non appellando" her, zumal der Artikel 19 des Krakauer Friedens, der eine Appellationsbestimmung enthielt, nur in denjenigen Ausnahmefällen von Albrecht die Zulassung von Appellationem an eine gemischte preußisch-polnische Kommission forderte, in denen der Herzog selbst als prozeßführende Partei auftrat. Als die Krone wenige Jahre nach Vertragsschluß versuchte, das polnische Jurisdiktionsrecht über die in den beiden Vertragspunkten klar gezogene Grenze hinaus zu erweitern und von Albrecht eine allgemeine Duldung von Appellationen an den Lehnsherrn zu fordern, führte Albrecht hiergegen den Wortlaut der Danziger Privilegienurkunde ins Feld. In ihr hatte Sigismund am 26. Mai 1526 in Danzig ${ }^{24}$ seinem Neffen alle vom Deutschen Orden überkommenen Rechte zugebilligt, ,exceptis his, quae nominatim in contractu concordiae" aufgeführt seien. Der Orden habe, so argumentierte Alb-

23 Zum folgenden Rechtsstreit näher: Dolezel, Lehnsverhältnis, $133 f$ f.

24 Dolezel, Staatsverträge, 54ff. 
recht treffend, die volle Appellationsfreiheit seit Kaiser Friedrichs II. Goldener Bulle von Rimini (1226) genossen; dem Herzog werde sie nur „nominatim”, das heißt, gemäß Artikel 19 Krakauer Friedens (nämlich bei Prozessen mit seinen eigenen Untertanen) eingeschränkt, generell jedoch, gemäß Artikel 20 des Krakauer Friedens und dem Wortlaut der Danziger Urkunde vollauf belassen. $\mathrm{Da} \beta$ sich Albrecht in diesem Falle als Rechtsnachfolger der Hochmeister fühlen durfte, konnte Adam Vetulani auf Grund einer eingehenden Analyse der genannten Belegstellen eindeutig feststellen ${ }^{25}$. Die Krone aber lehnte es seit 1532 beharrlich ab, diese Rechtslage anzuerkennen. Sie deduzierte, von der falschen These ausgehend, Appellationen an den Lehnsherren seien ganz allgemein ein lehnsrechtliches Postulat, ihre jurisdiktionellen Ansprüche aus der Krakauer Belehnung.

Auch Sigismund August begegnete dem genannten preußischen Rechtsanspruch, ebenso wie sein Vater, mit dem Argument, die Appellationsfreiheit habe der Herzog iure feudi eingebüßt, kam jedoch hierbei zu einer interessanten Feststellung, die Albrecht in der Frage der Königswahl seinem Lehnsherrn vergeblich hatte abringen wollen. Am 1. April 1552 mußten sich Albrechts Vertreter, Asverus von Brandt und Lukas David, von Sigismund August belehren lassen, daß der Herzog nicht als Rechtsnachfolger der Hochmeister anerkannt werden könne:

„Es sagen wohl Fürstlich Durchlaucht ${ }^{26}$, daß von dem Orden keine Appellation gestattet worden. Ihre Fürstlich Durchlaucht mußen dagegen aber wieder bekennen, daß es viel eine andere Gestalt mit dem Orden die Zeit gehabt, seintemal die Hoemeister niemals stadlich und gebuhrenderneise von den Königen von Polen die Lehen entpfangen und nurt allein mit dem Eide derselben Öberherrschaft erkannt".

\begin{abstract}
„Negat... in Prussia Dux ${ }^{27}$ a sententia Magistrorum in Prussia appellatum aliquando fuisse, sed fateatur oportet, longe aliam Magistrorum conditionem fuisse, qui nunquam solemnem investituram a ... Poloniae regibus accipiebant, sola iuris iurandi praestatione superioritatem illorum agnoscebantur".
\end{abstract}

25 Vetulani, Lenno, 199ff, besonders $217 \mathrm{ff}$.

26 Deutsche Fassung: 1552 April 1, [Petrikau], Antwort Sigismund Augusts, Ostpreußischer Foliant 102, fol. 242ff. Gedruckt bei Adalbert Bezzenberger: Die Berichte und Briefe des Rats und Gesandten Herzog Albrechts von Preußen, Asverus von Brandt, Band IV, Königsberg o. J., 468ff.

${ }_{27}$ Lateinische Fassung: Dogiel IV, 337 (irrtümlich 1553 datiert), hiernach auch Vetulani, Lenno, 254ff, jedoch mit anderer Fragestellung. 
$\mathrm{Daß}$ man die Auffassung, der Zweite Thorner Frieden habe 1466 kein Lehnsverhältnis begründet, in Königsberg, in der Umgebung des einstigen Ordenshochmeisters findet, mag nicht so sehr überraschen wie die Tatsache, daß sich dieselbe Auffassung auch auf polnischer Seite nachweisen läßt, und zwar nicht in einer unverbindlichen Verlautbarung, sondern in einem offiziellen responsum regium, innerhalb einer Quellengattung also von durchaus rechtlicher Relevanz, wenn man bedenkt, daß die meisten der zwischen dem Herzogtum und der Krone auftauchenden Streitfälle um die Interpretation des Krakauer Vertrags in der Form von königlichen responsa entschieden worden waren.

Die oben zitierte königliche Antwort - von Matthias Dogiel bereits 1764 publiziert, dennoch offenbar wenig bekannt - stellt vorerst ein Unikum dar. Die Frage, ob sich im Warschauer Archiwum Główne Akt Dawnych, etwa in den Beständen der Kronmetrik, weitere, vor allem ältere Rechtsaussagen dieser Art finden ließen, wäre ebenso interessant wie die möglicherweise nach stilistischen Kriterien $\mathrm{zu}$ treffende Feststellung, wem unter den königlichen Juristen des Jahres 1552 die eigentliche Autorschaft an dem responsum regium zukommt.

Freilich läßt sich mit dieser königlichen Antwort allein nicht letztlich die Frage lösen, wie die Krone das preußisch-polnische Rechtsverhältnis nach Thorn selbst interpretiert haben mag; das im doppelten Sinn des Wortes merkwürdige Aktenstück, wiewohl 86 Jahre nach dem Thorner Frieden und durchaus nicht zweckfrei entstanden, scheint jedoch für die künftige Diskussion um die Lehnsproblematik des Zweiten Thorner Friedens mehr zu sein als nur eine quantité négligeable ${ }^{28}$.

28 Abschluß des Manuskripts: Dezember 1974. 